\title{
UNIVERSIDADES INCLUSIVAS DE PAPEL. ANÁLISIS COMPARATIVO DE LA NORMATIVA DE DISCAPACIDAD DE LAS UNIVERSIDADES PÚBLICAS ANDALUZAS
}

\author{
Azahara Jiménez-Millán y María García-Cano Torrico \\ Universidad de Córdoba, España.m32jimia@uco.es; maria.garciacano@uco.es
}

\begin{abstract}
Resumen. El trabajo analiza la documentación institucional referida a la atención de personas con discapacidad en las universidades públicas andaluzas (España), como expresión de una educación de calidad equitativa e inclusiva. Para abordar esta finalidad, se emplea un enfoque cualitativo mediante Análisis Político del Discurso basado en el análisis de datos extraídos de las webs institucionales de las diez universidades públicas de Andalucía. Concretamente, se analiza la documentación institucional específica de discapacidad aprobada por sus Consejos de Gobierno con el fin de aportar elementos que evidencien cómo se materializa su responsabilidad social universitaria. Los resultados muestran heterogeneidad en la concreción de la política institucional de discapacidad, y aún incipiente la elaboración de planes que presentan un desarrollo comprometido mediante ejes, acciones y evaluación. En este sentido, la comparación de políticas ofrece claves importantes de buenas prácticas que pueden servir de referente entre instituciones.
\end{abstract}

Palabras clave: Universidad; Política Institucional; Inclusión; Comparación; Discapacidad. INCLUSIVE PAPER UNIVERSITIES. COMPARATIVE ANALYSIS OF THE DISABILITY
REGULATIONS OF ANDALUSIAN PUBLIC UNIVERSITIES

\begin{abstract}
The paper analyzes the institutional documentation referring to the Attention of people with disabilities in Andalusian public universities (Spain), as an expression of education of equitable and inclusive quality. To address this purpose, a qualitative approach is used through a Political Analysis of Discourse based on the analysis of data extracted from the institutional websites of the ten public universities in Andalusia. Specifically, the specific institutional documentation of disability approved by its Government Councils is analyzed in order to provide elements that demonstrate how its university social responsibility materializes. The results show heterogeneity in the concretion of the institutional disability policy; and it is incipient to elaborate plans that present a committed development through axes, actions, and evaluation. In this sense, the comparison of policies offers important keys to good practices that can serve as a reference among institutions.
\end{abstract}

Keywords: University; Institutional Policy; Inclusion; Comparison; Disability.

\section{INTRODUCCIÓN}

Desde el avance de la dimensión social del Proceso de Bolonia en la Conferencia de Londres (2017), el Espacio Europeo de Educación Superior (EEES) está encaminado hacia la construcción de una universidad diversa e inclusiva (Márquez y Sandoval, 2019). Sin embargo, el acceso, participación y logro de determinados grupos sociales en niveles educativos superiores sigue siendo meta a alcanzar (UNESCO, 2016), lo que queda evidenciado por la proclamación en la Agenda 2030 entre sus Objetivos de Desarrollo Sostenible: "garantizar una educación inclusiva, equitativa y de calidad y promover oportunidades de aprendizaje durante toda la vida para todos" (p. 1). 
El concepto de inclusión educativa en educación superior implica trabajar para eliminar actitudes y creencias negativas asociadas a raza, etnia, sexo, género, orientación sexual, estilo de vida, clase socio-económica, edad, lengua, religión, discapacidad y sus intersecciones, respetando su identidad individual y de grupo (Blessinger y Stefani, 2018). Sin embargo, en España los trabajos empíricos destacan mayor evidencia en el discurso y en el desarrollo normativo en relación con la protección de derechos y no discriminación de personas con discapacidad y la igualdad por razón de género (Márquez, Jiménez y Trabajo, 2018; Jiménez-Millán y García-Cano, 2018).

La educación superior inclusiva es un derecho universal reconocido por Tratados a nivel internacional cuya evolución ha quedado manifiesta a tenor de las distintas Convenciones Internacionales redactadas en las últimas décadas. Destacan, a pesar de las escasas alusiones al ámbito universitario, la Declaración de Salamanca (1994) con dos únicas referencias a las universidades (número 48, apartado C), la Declaración Mundial para la Educación Superior de la UNESCO (1998) y, posteriormente, la Convención Internacional sobre los Derechos de las Personas con Discapacidad (2006) cuyo artículo 24 apartado 2 establece "Los Estados Partes asegurarán que las personas con discapacidad no queden excluidas del sistema general de educación por motivos de discapacidad (...)" (p. 20654) y específicamente en el apartado 5 "Los Estados Partes asegurarán que las personas con discapacidad tengan acceso general a la educación superior (...)" (p. 20654) y en su artículo 27 recoge "el derecho de las personas con discapacidad a trabajar, en igualdad de condiciones (...) en un mercado y entorno laborales que sean abiertos, inclusivos y accesibles a las personas con discapacidad" (p. 20655)con la descripción de un conjunto de medidas preceptivas. Estos referentes son parte de los hitos normativos sobre el reconocimiento de las condiciones de igualdad de oportunidades y no discriminación de las personas con discapacidad.

En España, también la legislación específica ha caminado de manera progresiva desde el Plan Nacional para la Educación Especial, 1978; Ley de Integración Social de los Minusválidos, 1982; Real Decreto de Ordenación de la Educación Especial, 1985; Real Decreto de Ordenación de la Educación de los Alumnos con Necesidades Educativas Especiales, 1995 a la Ley de Igualdad de Oportunidades y No Discriminación de las Personas con Discapacidad (2003). 
En el contexto autonómico de Andalucía, el compromiso global de la promoción educativa y atención a la diversidad queda reflejado en normativas como la Ley 1/1999, de 32 de marzo, de Atención a las Personas con Discapacidad (LAPD), cuyo artículo 22 especifica las actuaciones concretas en la educación universitaria relativas a la elaboración de un censo, una unidad o servicio de atención, un plan de accesibilidad universal, adaptaciones o ajustes razonables, planes de formación, movilidad nacional e internacional y actuaciones a nivel de información, investigación y servicios relacionados. Además, la Ley 9/1999, de 18 de noviembre, de Solidaridad en la Educación y la concreción del I Plan de Acción Integral para las Personas con Discapacidad 2003-2006, cuya finalidad es a grandes rasgos, que todo estudiante, independientemente de sus circunstancias, pueda acceder y desarrollarse en la universidad sin obstáculos o barreras.

Este avance legislativo de la educación de los niños y niñas con discapacidad ha llevado aparejado el desarrollo de políticas educativas inclusivas $y$, por ende, un aumento progresivo de estudiantado universitario con discapacidad. En este sentido, un total de 22. 190 universitarios con discapacidad estudian en las universidades españolas (Universia, 2018). Este tránsito educativo de las personas con discapacidad hacia la universidad plantea a la institución superior un desafío ineludible que debe afrontar de forma coherente desde los principios de la educación inclusiva.

El desempeño y la atención a los estudiantes con discapacidad en la universidad en España está siendo abordado con trabajos empíricos desde distintas perspectivas. Por ejemplo, parte de las investigaciones sobre discapacidad en la universidad se centran en percepciones de los estudiantes que acceden (por ejemplo, Sánchez y Carrión, 2010) o cómo el profesorado desarrolla su desempeño docente al respecto (por ejemplo, Moriña, Sandoval y Carnerero 2020). Sin embargo, es escasa la literatura centrada en la comunidad universitaria, incluyendo a PAS y PDI con discapacidad, así como análisis de la respuesta institucional mediante normativas específicas en contextos universitarios locales. A este respecto, sí encontramos trabajos internacionales como el de Susan Iveson (2008) que realiza un análisis comparativo de planes de atención a la diversidad en cincuenta universidades en EE. UU. o Adriana Kezar (2007) que plantea si los campus en distintos momentos de institucionalización de la agenda de la diversidad cuentan con diferentes estrategias al respecto. 
En un trabajo anterior (Jiménez-Millán, 2020), se concluyó que el término inclusión es un concepto recurrente en la universidad y es utilizado de forma frecuente para identificar órganos institucionales encargados de la atención a las personas con discapacidad. A este respecto, Molina (2010) planteó la relación entre discapacidad, educación superior y política institucional y resaltó que, a pesar de un marco políticamente legitimado de igualdad de oportunidades e inclusión educativa para las personas con discapacidad, este objetivo político aun no alcanza suficientemente las instituciones con programas o reglamentos concretos. En esta línea, nos planteamos como pregunta de investigación ¿cuál es el marco y qué diferencias normativas se han plasmado en materia de discapacidad en las distintas universidades de Andalucía, España?

\section{OBJETIVOS}

Con el objeto de ampliar el conocimiento sobre la situación de las personas con discapacidad en la universidad, proponemos indagar en las webs institucionales de las diez universidades públicas andaluzas (Universidad de Almería [UAL], Universidad de Cádiz [UCA], Universidad de Córdoba [UCO], Universidad de Granada [UGR], Universidad de Huelva [UHU], Universidad Internacional de Andalucía [UNIA], Universidad de Jaén [UJA], Universidad de Málaga [UMA], Universidad Pablo de Olavide [UPO] y Universidad de Sevilla]) para realizar una aproximación a la normativa, reglamentación o programas políticos de acción específicos la comunidad universitaria con discapacidad en lo relativo a desarrollo reglamentario, concepto, ámbitos de aplicación, acciones y órganos responsable.

\section{METODOLOGÍA}

Este trabajo se plantea desde el Análisis Político de Discurso (APD) en el marco de la perspectiva cualitativa. Desde esta mirada, interesa el discurso político entendiendo la política como marco de acción, lugar de construcción, defensa de juicios y toma de decisiones "sobre qué es lo que hay que hacer en determinados eventos sociales y qué se articula en torno al por qué es necesario comprometernos con las acciones planteadas en los diversos discursos de política pública" (Palacios, Hidalgo, Cornejo y Suárez, 2019, p.14).

En el proceso metodológico, la primera fase corresponde con la búsqueda de las fuentes documentales en las páginas webs institucionales en el apartado que todas las universidades tienen destinadas a la atención a la diversidad/discapacidad; en la segunda fase, se valoraron los documentos encontrados y para ello, se creó una hoja de registro ad 
hoc (estimación/desestimación) para la recogida de todos los documentos institucionales cuya denominación incluyera el término diversidad y/o discapacidad obteniendo un total de 19. A estos documentos se les aplica como criterios de selección: (1) pertenecientes a las universidades públicas andaluzas, (2) elaborada por la propia universidad, (3) cuya denominación incluya el término discapacidad o diversidad, (4) aprobada por Consejo de Gobierno de la Universidad, y (5) vigente en el caso de encontrar varios. Tras desestimar 10 documentos no aprobados por Consejo de Gobierno, principalmente de tipo divulgativo, obtenemos un corpus documental de 9 documentos. Finalmente, la última fase y con el uso del software ATLAS.ti $v 8$, se realiza un análisis progresivo de los datos usando un sistema de categorías y códigos (Miles, Huberman \& Saldaña, 2014) identificando desarrollo reglamentario, concepto, ámbitos de aplicación, acciones y órganos responsable. El software nos permitió ordenar, establecer relaciones entre la normativa (derivada y dependiente) e identificar ejes y temáticas distintiva o común entre las distintas universidades.

\section{RESULTADOS}

La exploración de las webs institucionales de las universidades públicas andaluzas con normativa sobre inclusión nos ofrece 9 documentos específicos de discapacidad elaborados por las propias instituciones de educación superior (Tabla 1). De las 10 universidades, dos de ellas (UCA y UNIA) no elaboran ninguna reglamentación específica para este fin con los criterios indicados y hacen referencia a la normativa nacional y autonómica en materia de accesibilidad y Discapacidad sin especificar su concreción en la institución. Y, una tercera, la UAL, muestra un plan de actuación, pero sin referencia a su aprobación por el Consejo de Gobierno. Del resto de universidades, tres documentos corresponden a Reglamentos (UCO, UMA); cuatro, a Normativas (UGR, UJA, UHU) y dos son planes.

Estos últimos coinciden porque son los más actuales y para toda la comunidad universitaria de todos los revisados (UPO: II Plan de Accesibilidad e inclusión de la Diversidad Funcional 2019-2022, con 6 ejes, 16 objetivos y 38 acciones; y US: II Plan integral de Atención a las necesidades de apoyo para personas con discapacidad o con necesidad de apoyo por situación de salud sobrevenida, con 8 ejes, 17 objetivos y 73 acciones).

La mayoría de las instituciones realizan una presentación de la legislación universitaria, en mayor o menor medida, en referencia al ámbito internacional (ONU, Constitución Europea), nacional (Constitución Española, artículos 9.2,10,14 y 49, LOU, LOMLOU, Estatuto del 
Estudiante Universitario [EEU]) y autonómico (LAPD, TRLAU) con algunas particularidades como la UAL que además alude a las propuestas realizadas por parte del Comité que agrupa a las asociaciones de personas con discapacidad: "siguiendo las indicaciones del Comité Español de Representantes de Personas con Discapacidad (CERMI) en su Guía para la elaboración de un Plan de Atención al alumnado con Discapacidad en la Universidad, publicada en 2010" (p. 12). En este sentido, la mayoría de las universidades aluden a sus propios estatutos cuyos objetivos plantean esta necesidad.

En este desarrollo normativo se fundamentan la mayoría de las acciones y medidas que las universidades plantean como, por ejemplo, la exención de tasas (LOU, DA 24 ap.6), accesibilidad universal y diseño para todos (LOMLOU, DA7), obligación de la creación de un servicio de atención a la discapacidad (LOMLOU, DA24; EEU, art.65) o proporcionar recursos y adaptaciones curriculares (EEU, 12.b).

En relación con el ámbito de aplicación, el término elegido es diversidad funcional por dos universidades (UAL y UPO), cinco eligen discapacidad (UCO, UGR, UMA) o discapacidad funcional (UHU); dos, Necesidades Específicas de Apoyo Educativo (UGR, UJA) y una universidad selecciona el término Diversidad para el reglamento del Servicio que recibe el mismo nombre (UCO).

A este respecto, el $50 \%$ de las normativas objeto de estudio enuncian como destinatarios a todos los miembros de la comunidad universitaria, tres de ellas sólo identifican como destinados/as a estudiantes (UGR, UJA, UMA) y dos (UCO, UGR) han elaborado normativa específica para su personal (PDI y PAS).

El órgano responsable de las medidas que plantean en la mayoría de los casos se sitúa a nivel de Vicerrectorado de Estudiantes para aquellas normativas que son específicas de estudiantes; Vicerrectorado de Personal y/o gerente para aquellas que están destinadas específicamente para PDI y PAS o bajo el amparo del Vicerrectorado de Responsabilidad o políticas sociales (por ejemplo, en US de Servicios Sociales y Comunitarios) cuando el ámbito de aplicación es toda la comunidad universitaria.

Además, son los planes más integrales (UPO y US) los que presentan una mayor corresponsabilidad de vicerrectorados al detallar las acciones y responsables más directos en cada caso, por ejemplo, en áreas como investigación, internacionalización, digital o empleabilidad. 
Tabla 1. Normativa específica de discapacidad de las universidades públicas andaluzas

\begin{tabular}{|c|c|c|c|}
\hline Denominación & $\begin{array}{c}\text { Universidad/ } \\
\text { Aprobación Consejo } \\
\text { Gobierno }\end{array}$ & Ámbito de Aplicación & Órgano Responsable \\
\hline $\begin{array}{l}\text { Reglamento de Creación y Regulación } \\
\text { del Servicio de Atención a la } \\
\text { Diversidad de la Universidad de } \\
\text { Córdoba }\end{array}$ & $\begin{array}{l}\text { UCO } \\
25 \text { de mayo de } 2016\end{array}$ & $\begin{array}{l}\text { Diversidad } \\
\text { Comunidad } \\
\text { Universtaria }\end{array}$ & $\begin{array}{l}\text { Vicerrectorado ámbito } \\
\text { de atención a la } \\
\text { diversidad }\end{array}$ \\
\hline $\begin{array}{l}\text { Reglamento para facilitar la } \\
\text { incorporación e integración de personal } \\
\text { con discapacidad discapacidad a la } \\
\text { Universidad de Córdoba }\end{array}$ & 28 de abril de 2017 & $\begin{array}{l}\text { Discapacidad } \\
\text { Personal (PDI y PAS) }\end{array}$ & $\begin{array}{l}\text { Vicerrectorado y/o } \\
\text { gerente PDI y PAS }\end{array}$ \\
\hline $\begin{array}{l}\text { Normativa para la atención al } \\
\text { estudiantado con discapacidad y otras } \\
\text { necesidades específicas de apoyo } \\
\text { educativo }\end{array}$ & $\begin{array}{l}\text { UGR } \\
10 \text { de octubre de } 2016\end{array}$ & $\begin{array}{l}\text { Discapacidad y NEAE } \\
\text { Estudiantes }\end{array}$ & \\
\hline $\begin{array}{l}\text { Normativa para la adopción de } \\
\text { medidas de acción positiva y de } \\
\text { igualdad de oportunidades para el } \\
\text { personal de administración y servicios } \\
\text { y personal docente e investigador con } \\
\text { discapacidad }\end{array}$ & 10 de octubre de 2016 & $\begin{array}{l}\text { Discapacidad } \\
\text { Personal (PDI y PAS) }\end{array}$ & $\begin{array}{l}\text { Comisión de Medidas } \\
\text { de Acción positiva } \\
\text { para Personal de la } \\
\text { UGR con } \\
\text { Discapacidad }\end{array}$ \\
\hline $\begin{array}{l}\text { Normativa de igualdad de } \\
\text { oportunidades para personas con } \\
\text { discapacidad funcional }\end{array}$ & $\begin{array}{l}\text { UHU } \\
16 \text { de junio de } 2008\end{array}$ & $\begin{array}{l}\text { Diversidad Funcional } \\
\text { Comunidad } \\
\text { Universtaria }\end{array}$ & $\begin{array}{l}\text { Vicerrectorado } \\
\text { Estudiantes }\end{array}$ \\
\hline $\begin{array}{l}\text { Normativa que regula la atención a } \\
\text { estudiantes con necesidades } \\
\text { específicas de apoyo educativo en la } \\
\text { universidad de Jaén }\end{array}$ & $\begin{array}{l}\text { UJA } \\
27 \text { de junio de } 2016\end{array}$ & $\begin{array}{l}\text { NEAE } \\
\text { Estudiantes }\end{array}$ & $\begin{array}{l}\text { Vicerrectorado } \\
\text { Estudiantes, Inserción } \\
\text { laboral e } \\
\text { Internacionalización }\end{array}$ \\
\hline $\begin{array}{l}\text { Reglamento sobre Atención } \\
\text { Académica al Estudiante con } \\
\text { Discapacidad }\end{array}$ & $\begin{array}{l}\text { UMA } \\
20 \text { de diciembre de } \\
2012\end{array}$ & $\begin{array}{l}\text { Discapacidad } \\
\text { Estudiantes }\end{array}$ & $\begin{array}{l}\text { Vicerrectorado de } \\
\text { Estudiantes }>\text { Servicio } \\
\text { de Apoyo al } \\
\text { Alumnado con } \\
\text { Discapacidad }\end{array}$ \\
\hline $\begin{array}{l}\text { II Plan de Accesibilidad e inclusión de } \\
\text { la Diversidad Funcional 2019-2022 }\end{array}$ & $\begin{array}{l}\text { UPO } \\
21 \text { de mayo } 2019\end{array}$ & $\begin{array}{l}\text { Diversidad Funcional } \\
\text { Comunidad } \\
\text { Universtaria }\end{array}$ & \\
\hline $\begin{array}{l}\text { II Plan integral de Atención a las } \\
\text { necesidades de apoyo para personas } \\
\text { con discapacidad o con necesidad de } \\
\text { apoyo por situación de salud } \\
\text { sobrevenida }\end{array}$ & $\begin{array}{l}\text { US } \\
26 \text { de junio de } 2017\end{array}$ & $\begin{array}{l}\text { Personas con } \\
\text { discapacidad o con } \\
\text { necesidad de apoyo } \\
\text { por situación de salud } \\
\text { sobrevenida }\end{array}$ & $\begin{array}{l}\text { Vicerrectorado } \\
\text { Servicios Sociales y } \\
\text { Comunitarios }\end{array}$ \\
\hline
\end{tabular}

Elaboración propia

\section{CONCLUSIONES}

Los resultados evidencian que sólo dos de las diez universidades públicas andaluzas cuentan con un Plan de Inclusión o de Atención a las Personas con Discapacidad, que podría ser un instrumento más comprometido con la Inclusión puesto que, a diferencia de otras normativas, da muestra de un modelo más dinámico para su ejecución al estar organizado en ejes, objetivos y acciones, así como por presentar una evaluación y revisión. Además, el órgano responsable de la mayoría de los documentos se limita a Vicerrectorados y Servicios o Unidades de Atención a la Diversidad/Discapacidad siendo los Planes los que 
presentan unas actuaciones con mayor transversalidad al indicar mayor número de órganos responsables; por tanto, este es otro motivo para tomarlos como guía de buenas prácticas institucionales en el reto de la construcción de una universidad inclusiva.

La heterogeneidad de normativa institucional al respecto dificulta una sistematización que sí es posible en ámbitos como la igualdad entre mujeres y hombres como muestran trabajos como Hervías (2019) o como ocurre en planes de atención a la diversidad en Estados Unidos (Iverson, 2008). Nos encontramos, así, una compleja situación, en la que la mayoría de las universidades andaluzas deciden el desarrollo de una reglamentación que no concretan acciones, ni se vehiculan mecanismos de revisión, lo cual podría derivar en su no abordaje (Admed, 2019) o avance en dicha materia.

Los resultados son coincidentes en parte con otros estudios en otros contextos universitarios (Mejía Zapata, 2019) cuyo acceso no está garantizado y las barreras arquitectónicas siguen siendo un reto en la cobertura de los derechos constitucionales. No obstante, aporta la novedad de tomar como partida a la comunidad universitaria en su conjunto, de manera que se tenga por objeto no solo al estudiantado con discapacidad sino también al PAS y PDI.

En términos comparativos, parece que comienza a haber propuestas integrales que abarcan a toda la comunidad universitaria, con un periodo de ejecución y evaluación y con informe anual de memorias que avanza hacia la construcción de universidades más inclusivas. No obstante, este tipo de normativas son, de momento, una minoría; por ello, sería de interés que la academia tome como guía estos planteamientos para disponer de unas líneas presentes y futuras de actuación y actividades que puedan ser evaluadas de manera sistemática.

En estudios de estas características, sobre todo en términos comparados, el software utilizado es un instrumento al servicio de la interpretación empírica y teórica que puede servir para visualizar las evidencias, pero también ausencias, y las relaciones entre distintos contextos o casos estudiados.

Agradecimientos. Ministerio de Educación y Formación Profesional de España por las Ayudas para Formación del Profesorado Universitario (FPU-2018) y Ministerio de Economía, Industria y Competitividad de España, proyecto Ref. EDU2017-82862-R. 


\section{Referencias}

Admed, S. (2019). What's the use? United Kingdom: Duke University Press

Blessinger, P. \& Stefani, L. (2018). Inclusive Leadership in Higher Education: Inclusion as a Core Institutuinal Value. In L.Stefani, \& P. Blessinger, (2018). Inclusive Leadership in Higher Education. International Perspectives and Approaches. Routledge.

España, Convención Internacional de los Derechos de las Personas con Discapacidad, Boletín Oficial del Estado, 21 de abril de 2008, núm. 96

Hervías, V. (2019). El futuro de las políticas de igualdad de género en las universidades andaluzas. Un enfoque desde la política comparada. Edición no venal. Instituto Andaluz de la mujer.

Iverson (2008). Iverson, S. V. 2008. Capitalizing on Change: The Discursive Framing of Diversity in U.S. LandGrant Universities. Equity \& Excellence in Education 41(2): 182- 199. doi: 10.1080/10665680801972849

Jiménez-Millán, A. (2020). ¿Qué entiende la Universidad por Inclusión? Estudio comparado de las universidades públicas andaluzas. En Luque, I.y Mérida, J. (ed) Redes de Doctorandos. Vol. VI: La investigación del futuro. Universidad de Córdoba

Jiménez-Millán, A. \& García-Cano, M. (2019). Institutionalization proposals to diversity at University from the vision of leaders. In Carmo, M. (ed.). Education and new developments Vol. II, pp. 201-205. Lisboa: InSciencePress.

Kezar, A. J. (2007). Tools for a time and place: Phased leadership strategies to institutionalize a diversity agenda. The Review of Higher Education,30(4), 413-439.

Márquez Vázquez, C. \& Sandoval, M. (2019). Claves para promover la inclusión en la educación superior. En Carmen Márquez Vázquez (pp. 45-60) ¿Avanzamos hacia universidades más inclusivas? De la retórica a los hechos. Madrid: DYKINSON

Márquez, E., Jiménez, M. L., \& Trabajo, E. (2018). Análisis de las concepciones de la política universitaria sobre atención a la diversidad a través de documentos institucionales. Non-published Technical Report, R\&D Project EDU2017-82862-R.

Mejía Zapata, S.I. (2019). Diversidad funcional e inclusión en Instituciones de Educación Superior (IES) en Medellín, INTERDISCIPLINARIA, 2019, 36(2), 151-164

Miles, M.B, Huberman, A.M. \& Saldaña, J. (2014). Qualitative Data Analysis. A Methods Sourcebook. United States of America: Sage (Ed.3)

Molina, R. (2010). Educación superior para estudiantes con discapacidad. Revista de investigación, 34(70), 95115. https://dialnet.unirioja.es/servlet/articulo?codigo $=3427619$

Morilla, A., Aguirre, A. \& Doménech, A. (2019). Alumnado con Discapacidad en Educación Superior: ¿En qué, cómo $\mathrm{y}$ por qué se forma el profesorado universitario? Publicaciones, 49(3), 227-249. doi:10.30827/publicaciones.v49i3.11411

Morilla, A., Sandoval, M. \& Carnerero, F. (2020). Higher education inclusivity: when the disability enriches the university, Higher Education Research \& Development, doi:10.1080/07294360.2020.1712676

Palacios, D., Hidalgo, F., Cornejo, C., \& Suárez Monzón, N. (2019). Análisis Político de Discurso: Herramientas conceptuales y analíticas para el estudio crítico de políticas educativas en tiempos de reforma global. Archivos Analíticos de Políticas Educativas, 27(47) http://dx.doi.org/10.14507/epaa.27.4269

Sánchez, A., \& Carrión, J.J. (2010). Los estudiantes con discapacidad en la Universidad de Almería: Ideas y Actitudes sobre su integración Educativa y Social. European Journal of Education and Psychology, 3(2), 329-341.

UNESCO (2016). Training Tools for Curriculum Development. Reaching out to all Learning: a Resource Pack Supporting Inclusive Education. Geneva: International Bureau of Education-UNESCO. http://www.ibe.unesco.org/sites/default/files/resourses/ibe-crp-inclusiveeducation-2016_eng.pdf.

Universia (2018). Guía de Atención a la Discapacidad en la Universidad. Fundación Universia. https://www.fundacionuniversia.net/wp-content/uploads/2018/12/GUIA Atencion-a-la-discapacidad2018_ACCESIBLE.pdf 